\title{
A SD-IITFOWA OPERATOR AND TOPSIS BASED APPROACH FOR MAGDM PROBLEMS WITH INTUITIONISTIC TRAPEZOIDAL FUZZY NUMBERS
}

\author{
Jian WU \\ School of Economics and Management, Zhejiang Normal University, Box 62 at YingBinDaDao 688, \\ 321004 Jinhua, Zhejiang Province, China
}

Received 14 April 2012; accepted 04 August 2012

\begin{abstract}
The aim of this article is to investigate an approach to multiple attribute group decision making (MAGDM) problems in which the information about decision makers (DMs) weights is completely unknown in advance, the attributes are inter-dependent, and the attribute values take the form of intuitionistic trapezoidal fuzzy numbers. First, the concept of similarity degree (SD) for two intuitionistic trapezoidal fuzzy decision matrixes is defined, which measures the level of consensus between individual decision opinion and group decision opinion. Next, we develop some IITFOWA operators to aggregate intuitionistic trapezoidal fuzzy decision matrixes in MAGDM problems. In particular, we present the SD induced IITFOWA (SD-IITFOWA) operator, which induces the order of argument values by utilizing the similarity degree of decision makers. This operator aggregates individual opinion in such a way that more importance is placed on the most similarity one. Then, a SD-IITFOWA operator and TOPSIS method based approach is developed to solve the MAGDM problems with intuitionistic trapezoidal fuzzy numbers. Finally, the developed approach is used to select the right suppliers for a computer company.
\end{abstract}

Keywords: group decision making, multiple attribute decision making, induced intuitionistic trapezoidal fuzzy ordered weighted arithmetic (IITFOWA) operator, TOPSIS.

JEL Classification: C021, C441, D81, N55.

\section{Introduction}

In the process of multiple attribute group decision-making with uncertainty, decision makers (DMs) usually need to compare a finite set of alternatives $X=\left\{x_{1}, x_{2}, \ldots, x_{n}\right\}$ with respect to multiple attributes and construct individual decision matrix. Thereafter, the DMs need to aggregate the individual decision matrix into a collective one and rank the given alternatives.

Corresponding E-mail:

jyajian@163.com 
However, there usually arise situations of conflict and disagreement among preferences of DMs. Therefore, the consensus reaching process is necessary to obtain a final solution with a certain level of compatibility between DMs (Herrera-Viedma et al. 2002; Chiclana et al. 2003; Choudhury et al. 2006). So, one key issue in the theory of group judgments aggregation is how to choose the aggregation operator, which will affect the group DMs' compatibility degree (Wang, Fan 2007; Xu 2005, 2009; Dong et al. 2008; Wu et al.2009; Wu, Chiclana 2014a, 2014b).

However, many group decision making processes take place in an environment, where the information is not precisely known. Thus, the DMs may have vague knowledge about the preference degrees of one alternative over another, and cannot estimate alternative with an exact numerical value or an interval number. Atanassov (1986) developed the concept of intuitionistic fuzzy set (IFS) characterized by a membership function and a non-membership function, which is a generalization of the concept of fuzzy set (Zadeh 1965). The intuitionistic fuzzy set theory has been applied to many different fields, such as decision making (Li et al. 2009, 2010; Xu 2010, 2011; Liu 2011; Wang et al. 2009; Wei 2009, 2010a, 2010b, 2011; Wei, Zhao 2012; Wu, Chiclana 2012; Wu, Liu 2013; Wu et al. 2013), supplier selection (Boran et al. 2009; Ye 2010; Ashayeri et al. 2012), robot selection (Devi 2011), medical diagnosis (Szmidt, Kacprzyk 2004), investment option (Wei 2008), and logic programming (Mursaleen, Mohiuddine 2009; Zhang et al. 2009; Mohiuddine, Şevli 2011), artificial intelligence (Saadati et al. 2009). Later, Atanassov and Gargov (1989) introduced the notion of interval-valued intuitionistic in which membership function and non-membership function are interval-valued numbers. Recently, Nehi and Maleki (2005) proposed the definition of intuitionistic trapezoidal fuzzy numbers, which is characterized by a membership function and a non-membership function whose values are trapezoidal fuzzy numbers. Wang and Zhang (2009) developed the Hamming distance of intuitionistic trapezoidal fuzzy numbers and intuitionistic trapezoidal fuzzy weighted arithmetic averaging (ITFWAA) operator. Wei (2010c) proposed some arithmetic aggregation operators including intuitionistic trapezoidal fuzzy ordered weighted averaging (ITFOWA) operator and intuitionistic trapezoidal fuzzy hybrid aggregation (ITFHA) operator. Wu and Cao (2013) investigated the intuitionistic trapezoidal fuzzy ordered weighted geometric (ITFOWG) operator, induced intuitionistic trapezoidal fuzzy ordered weighted geometric (I-ITFOWG) operator and intuitionistic trapezoidal fuzzy hybrid geometric (ITFHG) operator. Zhang and Liu (2010) proposed a score function of triangular intuitionistic fuzzy numbers. Li (2010) introduced the concept of a ratio of the value index to the ambiguity index and developed a new methodology for ranking triangular intuitionistic fuzzy numbers. Ye (2011) developed a novel score function of trapezoidal intuitionistic fuzzy numbers and proposed the multi-attribute group decision making (MAGDM) problems. Nevertheless, these are few works focusing on the issue of improving group consistency degree under trapezoidal intuitionistic fuzzy environment. We still have the following questions:

(1) How to measure the level of consensus between individual decision opinion and group decision opinion under intuitionistic trapezoidal fuzzy environment?

(2) How to aggregate the individual intuitionistic trapezoidal fuzzy decision matrixes into a collective one in a reasonable way?

To resolve this problem, we define the concept of similarity degree (SD) for two intuitionistic trapezoidal fuzzy decision matrixes, which measures the level of consensus between individual decision opinion and group decision opinion. Then, we develop the SD induced 
intuitionistic trapezoidal fuzzy ordered weighted arithmetic (SD-IITFOWA) operator for aggregating group intuitionistic trapezoidal fuzzy information. This operator aggregates individual opinion in such a way that more importance is placed on the most similarity one. Then, combining the SD-IITFOWA operator and TOPSIS (Technique for Order Preference by Similarity to Ideal Solution) method (Hwang, Yoon 1981), this paper develops an approach to resolve the MAGDM problems in which the information about DM weights are completely unknown, the attributes are interdependent, and the attribute values take the form of intuitionistic trapezoidal fuzzy numbers.

In order to do so, the remainder of this paper is set out as follows. Section 1 introduces some basic concepts and some aggregation operators related to intuitionistic trapezoidal fuzzy sets. In Section 2, we shall present the IITFOWA operator and study the desirable properties. Section 3 defines the concept of similarity degree for two intuitionistic trapezoidal fuzzy decision matrixes and presents a particular case of IITFOWA operator: the SD-IITFOWA operator. Section 4 develops a SD-IITFOWA and TOPSIS method based approach to solve the MAGDM problems with intuitionistic trapezoidal fuzzy information. In Section 5, some illustrative examples are pointed out. Finally, in the last Section we draw our conclusions.

\section{Preliminaries}

In this section, some basic concepts and some aggregation operators related to intuitionistic fuzzy sets are introduced.

Definition 1 (Atanassov 1986). A generalized fuzzy set called intuitionistic fuzzy sets, shown as follows:

$$
A=\left\{<x, \mu_{A}(x), v_{A}(x)>\mid x \in X\right\},
$$

in which, $\mu_{A}$ means a membership function, and $v_{A}$ means a non-membership, with the condition $0 \leq \mu_{A}(x)+v_{A}(x) \leq 1, \mu_{A}(x), v_{A}(x) \in[0,1]$ for all $x \in X[1]$. For each $A$ in $X$, we can compute the intuitionistic index of the element $x$ in the set $A$, which is defined as follows:

$$
\pi_{A}(x)=1-u_{A}(x)-v_{A}(x) .
$$

Recently, Nehi and Maleki (2005) gave the definition of the intuitionistic trapezoidal fuzzy number. A prominent characteristic of the intuitionistic trapezoidal fuzzy set is that its membership values and non-membership values are trapezoidal fuzzy numbers.

Definition 2. An intuitionistic trapezoidal $A$ fuzzy number is denoted as $A=\left\langle\mu_{A}(x), v_{A}(x)\right\rangle=\left\langle\left(a_{1}, a_{2}, a_{3}, a_{4}\right),\left(b_{1}, b_{2}, b_{3}, b_{4}\right)\right\rangle$ in the set of real number $R$. Its membership function can be given as:

$$
\mu_{A}(x)= \begin{cases}\frac{x-a_{1}}{a_{2}-a_{1}}, & a_{1} \leq x \leq a_{2} \\ 1, & a_{2} \leq x \leq a_{3} \\ \frac{a_{4}-x}{a_{4}-a_{3}}, & a_{3} \leq x \leq a_{4} \\ 0, \text { others. }\end{cases}
$$


its non-membership function is:

$$
v_{A}(x)= \begin{cases}\frac{x-b_{1}}{b_{2}-b_{1}}, & b_{1} \leq x \leq b_{2} \\ 1, & b_{2} \leq x \leq b_{3} \\ \frac{b_{4}-x}{b_{4}-b_{3}}, & b_{3} \leq x \leq b_{4} \\ 0, \text { others }\end{cases}
$$

where $\left(a_{1}+a_{2}+a_{3}+a_{4}+b_{1}+b_{2}+b_{3}+b_{4}\right) / 4 \leq 1$.

To study the properties of ITFS, we define some operational laws as follows.

Definition 3. Let $A_{1}=\left\langle\left(a_{1}^{1}, a_{2}^{1}, a_{3}^{1}, a_{4}^{1}\right),\left(b_{1}^{1}, b_{2}^{1}, b_{3}^{1}, b_{4}^{1}\right)\right\rangle$ and $A_{2}=\left\langle\left(a_{1}^{2}, a_{2}^{2}, a_{3}^{2}, a_{4}^{2}\right),\left(b_{1}^{2}, b_{2}^{2}, b_{3}^{2}, b_{4}^{2}\right)\right\rangle$ be two intuitionistic trapezoidal fuzzy numbers, and $\lambda \geq 0$, then:

1) $A_{1} \cap A_{2}=\left\langle\left(\min \left(a_{1}^{1}, a_{1}^{2}\right), \min \left(a_{2}^{1}, a_{2}^{2}\right), \min \left(a_{3}^{1}, a_{3}^{2}\right), \min \left(a_{4}^{1}, a_{4}^{2}\right)\right)\right.$, $\left.\left(\max \left(b_{1}^{1}, b_{1}^{2}\right), \max \left(b_{2}^{1}, b_{2}^{2}\right), \max \left(b_{3}^{1}, b_{3}^{2}\right), \max \left(b_{4}^{1}, b_{4}^{2}\right)\right)\right\rangle ;$

2) $A_{1} \cup A_{2}=\left\langle\left(\max \left(a_{1}^{1}, a_{1}^{2}\right), \max \left(a_{2}^{1}, a_{2}^{2}\right), \max \left(a_{3}^{1}, a_{3}^{2}\right), \max \left(a_{4}^{1}, a_{4}^{2}\right)\right)\right.$, $\left.\left(\min \left(b_{1}^{1}, b_{1}^{2}\right), \min \left(b_{2}^{1}, b_{2}^{2}\right), \min \left(b_{3}^{1}, b_{3}^{2}\right), \min \left(b_{4}^{1}, b_{4}^{2}\right)\right)\right\rangle ;$

3) $A_{1} \oplus A_{2}=\left\langle\left(a_{1}^{1}+a_{1}^{2}-a_{1}^{1} a_{1}^{2}, a_{2}^{1}+a_{2}^{2}-a_{2}^{1} a_{2}^{2}, a_{3}^{1}+a_{3}^{2}-a_{3}^{1} a_{3}^{2}, a_{4}^{1}+a_{4}^{2}-a_{4}^{1} a_{4}^{2}\right)\right.$, $\left.\left(b_{1}^{1} b_{1}^{2}, b_{2}^{1} b_{2}^{2}, b_{3}^{1} b_{3}^{2}, b_{4}^{1} b_{4}^{2}\right)\right\rangle$;

4) $A_{1} \otimes A_{2}=\left\langle\left(a_{1}^{1} a_{1}^{2}, a_{2}^{1} a_{2}^{2}, a_{3}^{1} a_{3}^{2}, a_{4}^{1} a_{4}^{2}\right)\right.$,

$$
\left.\left(b_{1}^{1}+b_{1}^{2}-b_{1}^{1} b_{1}^{2}, b_{2}^{1}+b_{2}^{2}-b_{2}^{1} b_{2}^{2}, b_{3}^{1}+b_{3}^{2}-b_{3}^{1} b_{3}^{2}, b_{4}^{1}+b_{4}^{2}-b_{4}^{1} b_{4}^{2}\right)\right\rangle ;
$$

5) $\lambda A_{1}=\left\langle\left(1-\left(1-a_{1}^{1}\right)^{\lambda}, 1-\left(1-a_{2}^{1}\right)^{\lambda}, 1-\left(1-a_{3}^{1}\right)^{\lambda}, 1-\left(1-a_{4}^{1}\right)^{\lambda}\right),\left(\left(b_{1}^{1}\right)^{\lambda},\left(b_{2}^{1}\right)^{\lambda},\left(b_{3}^{1}\right)^{\lambda},\left(b_{4}^{1}\right)^{\lambda}\right)\right\rangle$;

6) $A_{1}^{\lambda}=\left\langle\left(\left(a_{1}^{1}\right)^{\lambda},\left(a_{2}^{1}\right)^{\lambda},\left(a_{3}^{1}\right)^{\lambda},\left(a_{4}^{1}\right)^{\lambda}\right),\left(1-\left(1-b_{1}^{1}\right)^{\lambda}, 1-\left(1-b_{2}^{1}\right)^{\lambda}, 1-\left(1-b_{3}^{1}\right)^{\lambda}, 1-\left(1-b_{4}^{1}\right)^{\lambda}\right)\right\rangle$.

In the following, we prove that the operational results by same laws in Definition 3 are also intuitionistic trapezoidal fuzzy sets.

Theorem 1. Let $A_{1}=\left\langle\left(a_{1}^{1}, a_{2}^{1}, a_{3}^{1}, a_{4}^{1}\right),\left(b_{1}^{1}, b_{2}^{1}, b_{3}^{1}, b_{4}^{1}\right)\right\rangle$ and $A_{2}=\left\langle\left(a_{1}^{2}, a_{2}^{2}, a_{3}^{2}, a_{4}^{2}\right),\left(b_{1}^{2}, b_{2}^{2}, b_{3}^{2}, b_{4}^{2}\right)\right\rangle$ be two intuitionistic trapezoidal fuzzy numbers, then both $A_{1} \oplus A_{2}$ and $\lambda A_{1}$ are also intuitionistic trapezoidal fuzzy numbers.

Proof. From Definition 3, we obtain:

$$
\begin{aligned}
A_{1} \oplus A_{2}= & \left\langle\left(a_{1}^{1}+a_{1}^{2}-a_{1}^{1} a_{1}^{2}, a_{2}^{1}+a_{2}^{2}-a_{2}^{1} a_{2}^{2}, a_{3}^{1}+a_{3}^{2}-a_{3}^{1} a_{3}^{2}, a_{4}^{1}+a_{4}^{2}-a_{4}^{1} a_{4}^{2}\right),\right. \\
& \left.\left(b_{1}^{1} b_{1}^{2}, b_{2}^{1} b_{2}^{2}, b_{3}^{1} b_{3}^{2}, b_{4}^{1} b_{4}^{2}\right)\right\rangle .
\end{aligned}
$$


Since:

$$
\begin{aligned}
& a_{1}^{1}+a_{1}^{2}-a_{1}^{1} a_{1}^{2}+b_{1}^{1} b_{1}^{2} \leq\left(1-a_{1}^{1}\right)\left(1-a_{1}^{2}\right)+a_{1}^{1}+a_{1}^{2}-a_{1}^{1} a_{1}^{2}=1, \\
& a_{2}^{1}+a_{2}^{2}-a_{2}^{1} a_{2}^{2}+b_{2}^{1} b_{2}^{2} \leq\left(1-a_{2}^{1}\right)\left(1-a_{2}^{2}\right)+a_{2}^{1}+a_{2}^{2}-a_{2}^{1} a_{2}^{2}=1, \\
& a_{3}^{1}+a_{3}^{2}-a_{3}^{1} a_{3}^{2}+b_{3}^{1} b_{3}^{2} \leq\left(1-a_{3}^{1}\right)\left(1-a_{3}^{2}\right)+a_{3}^{1}+a_{3}^{2}-a_{3}^{1} a_{3}^{2}=1, \\
& a_{4}^{1}+a_{4}^{2}-a_{4}^{1} a_{4}^{2}+b_{4}^{1} b_{4}^{2} \leq\left(1-a_{4}^{1}\right)\left(1-a_{4}^{2}\right)+a_{4}^{1}+a_{4}^{2}-a_{4}^{1} a_{4}^{2}=1 .
\end{aligned}
$$

Then:

$\left(a_{1}^{1}+a_{1}^{2}-a_{1}^{1} a_{1}^{2}+a_{2}^{1}+a_{2}^{2}-a_{2}^{1} a_{2}^{2}+a_{3}^{1}+a_{3}^{2}-a_{3}^{1} a_{3}^{2}+a_{4}^{1}+a_{4}^{2}-a_{4}^{1} a_{4}^{2}+b_{1}^{1} b_{1}^{2}+b_{2}^{1} b_{2}^{2}+b_{3}^{1} b_{3}^{2}+b_{4}^{1} b_{4}^{2}\right) \leq 1$.

Thus $A_{1} \oplus A_{2}$ is an intuitionistic trapezoidal fuzzy number.

From Definition 3, we have:

$$
\lambda A_{1}=\left\langle\left(1-\left(1-a_{1}^{1}\right)^{\lambda}, 1-\left(1-a_{2}^{1}\right)^{\lambda}, 1-\left(1-a_{3}^{1}\right)^{\lambda}, 1-\left(1-a_{4}^{1}\right)^{\lambda}\right),\left(\left(b_{1}^{1}\right)^{\lambda},\left(b_{2}^{1}\right)^{\lambda},\left(b_{3}^{1}\right)^{\lambda},\left(b_{4}^{1}\right)^{\lambda}\right)\right\rangle .
$$

Since:

$$
\begin{aligned}
& 1-\left(1-a_{1}^{1}\right)^{\lambda} \leq\left(a_{1}^{1}\right)^{\lambda}, 1-\left(1-a_{2}^{1}\right)^{\lambda} \leq\left(a_{2}^{1}\right)^{\lambda}, \\
& 1-\left(1-a_{3}^{1}\right)^{\lambda} \leq\left(a_{3}^{1}\right)^{\lambda}, 1-\left(1-a_{4}^{1}\right)^{\lambda} \leq\left(a_{4}^{1}\right)^{\lambda} .
\end{aligned}
$$

Then:

$$
\begin{gathered}
\left(1-\left(1-a_{1}^{1}\right)^{\lambda}+1-\left(1-a_{2}^{1}\right)^{\lambda}+1-\left(1-a_{3}^{1}\right)^{\lambda}+1-\left(1-a_{4}^{1}\right)^{\lambda}+\left(b_{1}^{1}\right)^{\lambda}+\left(b_{2}^{1}\right)^{\lambda}+\left(b_{3}^{1}\right)^{\lambda}+\left(b_{4}^{1}\right)^{\lambda}\right) / 4 \leq \\
\left(\left(a_{1}^{1}\right)^{\lambda}+\left(a_{2}^{1}\right)^{\lambda}+\left(a_{3}^{1}\right)^{\lambda}+\left(a_{4}^{1}\right)^{\lambda}+\left(b_{1}^{1}\right)^{\lambda}+\left(b_{2}^{1}\right)^{\lambda}+\left(b_{3}^{1}\right)^{\lambda}+\left(b_{4}^{1}\right)^{\lambda}\right) / 4 \leq \\
\left(a_{1}+a_{2}+a_{3}+a_{4}+b_{1}+b_{2}+b_{3}+b_{4}\right) / 4 \leq 1 .
\end{gathered}
$$

Thus $\lambda A_{1}$ is also an intuitionistic trapezoidal fuzzy number, which completes the proof of Theorem 1 .

Theorem 2. Let $A_{1}=\left\langle\left(a_{1}^{1}, a_{2}^{1}, a_{3}^{1}, a_{4}^{1}\right),\left(b_{1}^{1}, b_{2}^{1}, b_{3}^{1}, b_{4}^{1}\right)\right\rangle$ and $A_{2}=\left\langle\left(a_{1}^{2}, a_{2}^{2}, a_{3}^{1}, a_{4}^{1}\right),\left(b_{1}^{2}, b_{2}^{2}, b_{3}^{2}, b_{4}^{2}\right)\right\rangle$ be two intuitionistic trapezoidal fuzzy numbers, $\lambda, \lambda_{1}, \lambda_{2}>0$, then:

1) $A_{1} \oplus A_{2}=A_{2} \oplus A_{1}$;

2) $\lambda\left(A_{1} \oplus A_{2}\right)=\lambda A_{2} \oplus \lambda A_{1}$;

3) $\lambda_{1} A_{1} \oplus \lambda_{2} A_{1}=\left(\lambda_{1}+\lambda_{2}\right) A_{1}$.

\section{Proof:}

1) This result is obvious.

2) Applying the operational law 2) in Definition 3, we have:

$$
\begin{aligned}
A_{1} \oplus A_{2}= & \left\langle\left(a_{1}^{1}+a_{1}^{2}-a_{1}^{1} a_{1}^{2}, a_{2}^{1}+a_{2}^{2}-a_{2}^{1} a_{2}^{2}, a_{3}^{1}+a_{3}^{2}-a_{3}^{1} a_{3}^{2}, a_{4}^{1}+a_{4}^{2}-a_{4}^{1} a_{4}^{2}\right),\right. \\
& \left.\left(b_{1}^{1} b_{1}^{2}, b_{2}^{1} b_{2}^{2}, b_{3}^{1} b_{3}^{2}, b_{4}^{1} b_{4}^{2}\right)\right\rangle .
\end{aligned}
$$


Then, by the operational law 5) in Definition 3, it follows that:

$$
\begin{gathered}
\lambda\left(A_{1} \oplus A_{2}\right)=\left\langle\left( 1-\left[\left(1-a_{1}^{1}\right)\left(1-a_{1}^{2}\right)\right]^{\lambda}, 1-\left[\left(1-a_{2}^{1}\right)\left(1-a_{2}^{2}\right)\right]^{\lambda}, 1-\left[\left(1-a_{3}^{1}\right)\left(1-a_{3}^{2}\right)\right]^{\lambda},\right.\right. \\
\left.\left.1-\left[\left(1-a_{4}^{1}\right)\left(1-a_{4}^{2}\right)\right]^{\lambda}\right),\left(\left(b_{1}^{1} b_{1}^{2}\right)^{\lambda},\left(b_{2}^{1} b_{2}^{2}\right)^{\lambda},\left(b_{3}^{1} b_{3}^{2}\right)^{\lambda},\left(b_{4}^{1} b_{4}^{2}\right)^{\lambda}\right)\right\rangle .
\end{gathered}
$$

Also since:

$$
\begin{aligned}
& \lambda A_{1}=\left\langle\left(1-\left(1-a_{1}^{1}\right)^{\lambda}, 1-\left(1-a_{2}^{1}\right)^{\lambda}, 1-\left(1-a_{3}^{1}\right)^{\lambda}, 1-\left(1-a_{4}^{1}\right)^{\lambda}\right),\left(\left(b_{1}^{1}\right)^{\lambda},\left(b_{2}^{1}\right)^{\lambda},\left(b_{3}^{1}\right)^{\lambda},\left(b_{4}^{1}\right)^{\lambda}\right)\right\rangle ; \\
& \lambda A_{2}=\left\langle\left(1-\left(1-a_{1}^{2}\right)^{\lambda}, 1-\left(1-a_{2}^{2}\right)^{\lambda}, 1-\left(1-a_{3}^{2}\right)^{\lambda}, 1-\left(1-a_{4}^{2}\right)^{\lambda}\right),\left(\left(b_{1}^{2}\right)^{\lambda},\left(b_{2}^{2}\right)^{\lambda},\left(b_{3}^{2}\right)^{\lambda},\left(b_{4}^{2}\right)^{\lambda}\right)\right\rangle .
\end{aligned}
$$

Then we have:

$$
\begin{gathered}
\lambda A_{1} \oplus \lambda A_{2}=\left\langle\left( 1-\left[\left(1-a_{1}^{1}\right)\left(1-a_{1}^{2}\right)\right]^{\lambda}, 1-\left[\left(1-a_{2}^{1}\right)\left(1-a_{2}^{2}\right)\right]^{\lambda}, 1-\left[\left(1-a_{3}^{1}\right)\left(1-a_{3}^{2}\right)\right]^{\lambda},\right.\right. \\
\left.\left.1-\left[\left(1-a_{4}^{1}\right)\left(1-a_{4}^{2}\right)\right]^{\lambda}\right),\left(\left(b_{1}^{1} b_{1}^{2}\right)^{\lambda},\left(b_{2}^{1} b_{2}^{2}\right)^{\lambda},\left(b_{3}^{1} b_{3}^{2}\right)^{\lambda},\left(b_{4}^{1} b_{4}^{2}\right)^{\lambda}\right)\right\rangle .
\end{gathered}
$$

Hence:

$$
\lambda\left(A_{1} \oplus A_{2}\right)=\lambda A_{1} \oplus \lambda A_{2} .
$$

3) By the operational law 5) in Definition 3, we obtain:

$$
\begin{aligned}
& \lambda_{1} A_{1}=\left\langle\left(1-\left(1-a_{1}^{1}\right)^{\lambda_{1}}, 1-\left(1-a_{2}^{1}\right)^{\lambda_{1}}, 1-\left(1-a_{3}^{1}\right)^{\lambda_{1}}, 1-\left(1-a_{4}^{1}\right)^{\lambda_{1}}\right),\left(\left(b_{1}^{1}\right)^{\lambda_{1}},\left(b_{2}^{1}\right)^{\lambda_{1}},\left(b_{3}^{1}\right)^{\lambda_{1}},\left(b_{4}^{1}\right)^{\lambda_{1}}\right)\right\rangle ; \\
& \lambda_{2} A_{1}=\left\langle\left(1-\left(1-a_{1}^{1}\right)^{\lambda_{2}}, 1-\left(1-a_{2}^{1}\right)^{\lambda_{2}}, 1-\left(1-a_{3}^{1}\right)^{\lambda_{2}}, 1-\left(1-a_{4}^{1}\right)^{\lambda_{2}}\right),\left(\left(b_{1}^{1}\right)^{\lambda_{2}},\left(b_{2}^{1}\right)^{\lambda_{2}},\left(b_{3}^{1}\right)^{\lambda_{2}},\left(b_{4}^{1}\right)^{\lambda_{2}}\right)\right\rangle .
\end{aligned}
$$

Then:

$$
\begin{aligned}
\lambda_{1} A_{1} \oplus \lambda_{2} A_{1}= & \left\langle\left( 1-\left(1-a_{1}^{1}\right)^{\lambda_{1}}\left(1-a_{1}^{1}\right)^{\lambda_{2}}, 1-\left(1-a_{2}^{1}\right)^{\lambda_{1}}\left(1-a_{2}^{1}\right)^{\lambda_{2}, 1-}\left(1-a_{3}^{1}\right)^{\lambda_{1}}\left(1-a_{3}^{1}\right)^{\lambda_{2}},\right.\right. \\
& \left.1-\left(1-a_{4}^{1}\right)^{\lambda_{1}}\left(1-a_{4}^{1}\right)^{\lambda_{2}},\left(\left(b_{1}^{1}\right)^{\lambda_{1}}\left(b_{1}^{1}\right)^{\lambda_{2}},\left(b_{2}^{1}\right)^{\lambda_{1}}\left(b_{2}^{1}\right)^{\lambda_{2}},\left(b_{3}^{1}\right)^{\lambda_{1}}\left(b_{3}^{1}\right)^{\lambda_{2}},\left(b_{4}^{1}\right)^{\lambda_{1}}\left(b_{4}^{1}\right)^{\lambda_{2}}\right)\right\rangle= \\
& \left\langle\left( 1-\left(1-a_{1}^{1}\right)^{\lambda_{1}+\lambda_{2}}, 1-\left(1-a_{2}^{1}\right)^{\lambda_{1}+\lambda_{2}}, 1-\left(1-a_{3}^{1}\right)^{\lambda_{1}+\lambda_{2}}, 1-\left(1-a_{4}^{1}\right)^{\lambda_{1}+\lambda_{2},},\right.\right. \\
& \left.\left(\left(b_{1}^{1}\right)^{\lambda_{1}+\lambda_{2}},\left(b_{2}^{1}\right)^{\lambda_{1}+\lambda_{2}},\left(b_{3}^{1}\right)^{\lambda_{1}+\lambda_{2}},\left(b_{4}^{1}\right)^{\lambda_{1}+\lambda_{2}}\right)\right\rangle=\left(\lambda_{1}+\lambda_{2}\right) A_{1} .
\end{aligned}
$$

which completes the proof of Theorem 2.

\section{Induced intuitionistic trapezoidal fuzzy ordered weighted averaging (IITFO-WA) operator}

The Induced Ordered Weighted Averaging (IOWA) was introduced by Yager and Filev (1999), which has been widely used in GDM problems. Chiclana et al. (2007) presented aparticular IOWA operator: the Consistency IOWA (C-IOWA) operator, which applied the ordering of the argument values based upon the consistency of the information sources. It is worthy of being mentioned that the C-IOWA operator aggregates individual preferences in such a way that more importance is placed on the most consistent one. Recently, Merigó et al. (Merigó, 
Casanovas 2010, 2011; Merigó, Gil-Lafuente 2011) developed some families of uncertain and fuzzy induced operators for group decision making. In $\mathrm{Wu}, \mathrm{Cao}$ (2011), we proved that the C-IOWA operator can improve the group consistency. In this section, we shall develop the IITFOWA operator for aggregating intuitionistic trapezoidal fuzzy numbers and study its desirable properties.

Definition 4. Let $A_{j}=\left\langle\left(a_{1}^{j}, a_{2}^{j}, a_{3}^{j}, a_{4}^{j}\right),\left(b_{1}^{j}, b_{2}^{j}, b_{3}^{j}, b_{4}^{j}\right)\right\rangle(j=1,2, \ldots, n)$ be a collection of intuitionistic fuzzy values, and then the induced intuitionistic trapezoidal fuzzy ordered weighted averaging (IITFOWA) operator is defined as follows:

$$
\begin{array}{r}
\operatorname{IITFOWA}_{w}\left(\left\langle u_{1}, A_{1}\right\rangle, \ldots,\left\langle u_{n}, A_{n}\right\rangle\right)=\left\langle\left( 1-\prod_{j=1}^{n}\left(1-a_{1}^{\sigma(j)}\right)^{w_{j}}, 1-\prod_{j=1}^{n}\left(1-a_{2}^{\sigma(j)}\right)^{w_{j}}, 1-\prod_{j=1}^{n}\left(1-a_{3}^{\sigma(j)}\right)^{w_{j}},\right.\right. \\
\left.\left.1-\prod_{j=1}^{n}\left(1-a_{4}^{\sigma(j)}\right)^{w_{j}}\right),\left(\prod_{j=1}^{n}\left(b_{1}^{\sigma(j)}\right)^{w_{j}}, \prod_{j=1}^{n}\left(b_{2}^{\sigma(j)}\right)^{w_{j}}, \prod_{j=1}^{n}\left(b_{3}^{\sigma(j)}\right)^{w_{j}}, \prod_{j=1}^{n}\left(b_{4}^{\sigma(j)}\right)^{w_{j}}\right)\right\rangle,
\end{array}
$$

where $w=\left(w_{1}, w_{2}, \ldots, w_{n}\right)^{T}$ be the weighting vector of $\tilde{a}_{j}(j=1,2, \ldots, n)$, where $\omega_{j}>0$, $\sum_{j=1}^{n} w_{j}=1, A_{\sigma(j)}=\left\langle\left(a_{1}^{\sigma(j)}, a_{2}^{\sigma(j)}, a_{3}^{\sigma(j)}, a_{4}^{\sigma(j)}\right),\left(b_{1}^{\sigma(j)}, b_{2}^{\sigma(j)}, b_{3}^{\sigma(j)}, b_{4}^{\sigma(j)}\right)\right\rangle$ is the $A_{i}$ value of the IFOWA pair $\left\langle u_{i}, A_{i}\right\rangle$ having the $j$-th largest $u_{i}\left(u_{i} \in[0,1]\right)$, and $u_{i} \operatorname{in}\left\langle u_{i}, A_{i}\right\rangle$ is referred to as the order inducing variable and $A_{i}$ as the intuitionistic trapezoidal fuzzy values.

The IITFOWA operator satisfies the following properties:

Theorem 3. (Commutativity).

$\operatorname{IITFOWA}_{w}\left(\left\langle u_{1}, A_{1}\right\rangle,\left\langle u_{2}, A_{2}\right\rangle, \ldots,\left\langle u_{n}, A_{n}\right\rangle\right)=\operatorname{IITFOWA}_{w}\left(\left\langle u_{1}, A_{1}^{\prime}\right\rangle,\left\langle u_{2}, A_{2}^{\prime}\right\rangle, \ldots,\left\langle u_{n}, A_{n}^{\prime}\right\rangle\right)$, where $\left(\left\langle u_{1}, A_{1}^{\prime}\right\rangle,\left\langle u_{2}, A_{2}^{\prime}\right\rangle, \ldots,\left\langle u_{n}, A_{n}^{\prime}\right\rangle\right)$ is any permutation of $\left(\left\langle u_{1}, A_{1}\right\rangle,\left\langle u_{2}, A_{2}\right\rangle, \ldots,\left\langle u_{n}, A_{n}\right\rangle\right)$.

Proof. Let

$$
\begin{aligned}
& \operatorname{IITFOWA}_{w}\left(\left\langle u_{1}, A_{1}\right\rangle,\left\langle u_{2}, A_{2}\right\rangle, \ldots,\left\langle u_{n}, A_{n}\right\rangle\right)=\sum_{j=1}^{n} A_{\sigma(j)} w_{j}, \\
& \operatorname{IITFOWA}_{w}\left(\left\langle u_{1}, A_{1}^{\prime}\right\rangle,\left\langle u_{2}, A_{2}^{\prime}\right\rangle, \ldots,\left\langle u_{n}, A_{n}^{\prime}\right\rangle\right)=\sum_{j=1}^{n} A_{\sigma(j)}^{\prime} w_{j} .
\end{aligned}
$$

Since $\left(\left\langle u_{1}, A_{1}^{\prime}\right\rangle,\left\langle u_{2}, A_{2}^{\prime}\right\rangle, \ldots,\left\langle u_{n}, A_{n}^{\prime}\right\rangle\right)$ is any permutation of $\left(\left\langle u_{1}, A_{1}\right\rangle,\left\langle u_{2}, A_{2}\right\rangle, \ldots,\left\langle u_{n}, A_{n}\right\rangle\right)$, we can obtain $A_{\sigma(j)}=A_{\sigma(j)}^{\prime}(j=1,2, \ldots, n)$ and then have:

$\operatorname{IITFOWA}_{w}\left(\left\langle u_{1}, A_{1}\right\rangle,\left\langle u_{2}, A_{2}\right\rangle, \ldots,\left\langle u_{n}, A_{n}\right\rangle\right)=\operatorname{IITFOWA}_{w}\left(\left\langle u_{1}, A_{1}^{\prime}\right\rangle,\left\langle u_{2}, A_{2}^{\prime}\right\rangle, \ldots,\left\langle u_{n}, A_{n}^{\prime}\right\rangle\right)$.

Theorem 4. (Idempotency).

If $A_{j}=A$ for all $j$, then $\operatorname{IITFOWA}_{w}\left(\left\langle u_{1}, A_{1}\right\rangle,\left\langle u_{2}, A_{2}\right\rangle, \ldots,\left\langle u_{n}, A_{n}\right\rangle\right)=A$.

Proof. Since $A_{j}=A$ for all $j$, we have:

$\operatorname{IITFOWA}_{w}\left(\left\langle u_{1}, A_{1}\right\rangle, \ldots,\left\langle u_{n}, A_{n}\right\rangle\right)=\left\langle\left(a_{1}, a_{2}, a_{3}, a_{4}\right),\left(b_{1}, b_{2}, b_{3}, b_{4}\right)\right\rangle=A$. 
Theorem 5. (Monotonicity).

If $A_{j} \leq A_{j}^{\prime}$ forall $j$, then $\operatorname{IITFOWA}_{w}\left(\left\langle u_{1}, A_{1}\right\rangle,\left\langle u_{2}, A_{2}\right\rangle, \ldots,\left\langle u_{n}, A_{n}\right\rangle\right) \leq \operatorname{IITFOWA}_{w}\left(\left\langle u_{1}, A_{1}^{\prime}\right\rangle\right.$, $\left.\left\langle u_{2}, A_{2}^{\prime}\right\rangle, \ldots,\left\langle u_{n}, A_{n}^{\prime}\right\rangle\right)$.

Proof. Let

$$
\begin{aligned}
& \operatorname{IITFOWA}_{w}\left(\left\langle u_{1}, A_{1}\right\rangle,\left\langle u_{2}, A_{2}\right\rangle, \ldots,\left\langle u_{n}, A_{n}\right\rangle\right)=\sum_{j=1}^{n} A_{\sigma(j)} w_{j}, \\
& \operatorname{IITFOWA}_{w}\left(\left\langle u_{1}, A_{1}^{\prime}\right\rangle,\left\langle u_{2}, A_{2}^{\prime}\right\rangle, \ldots,\left\langle u_{n}, A_{n}^{\prime}\right\rangle\right)=\sum_{j=1}^{n} A_{\sigma(j)}^{\prime} w_{j} .
\end{aligned}
$$

Since $A_{j} \leq A_{j}^{\prime}$ for $j$, it follows that $A_{\sigma(j)} \leq A_{\sigma(j)}^{\prime}$, then IITFOWA ${ }_{w}\left(\left\langle u_{1}, A_{1}\right\rangle,\left\langle u_{2}, A_{2}\right\rangle, \ldots\right.$, $\left.\left\langle u_{n}, A_{n}\right\rangle\right) \leq \operatorname{IITFOWA}_{w}\left(\left\langle u_{1}, A_{1}^{\prime}\right\rangle,\left\langle u_{2}, A_{2}^{\prime}\right\rangle, \ldots,\left\langle u_{n}, A_{n}^{\prime}\right\rangle\right)$.

Theorem 6. Let $A_{j}=\left\langle\left(a_{1}^{j}, a_{2}^{j}, a_{3}^{j}, a_{4}^{j}\right),\left(b_{1}^{j}, b_{2}^{j}, b_{3}^{j}, b_{4}^{j}\right)\right\rangle(j=1,2, \ldots, n)$ be a collection of intuitionistic fuzzy values and $w=\left(w_{1}, w_{2}, \ldots, w_{n}\right)^{T}$ be the weighting vector of IITFOWA operator, where $w_{j}>0, \sum_{j=1}^{n} w_{j}=1$. Then we have the two following cases:

1) If $w=(1,0, \ldots, 0)^{T}$, then the IITFOWA operator is reduced to the maximum operator:

$$
\operatorname{IITFOWA}_{w}\left(\left\langle\tilde{a}_{1}, \tilde{a}_{1}\right\rangle,\left\langle\tilde{a}_{2}, \tilde{a}_{2}\right\rangle, \ldots,\left\langle\tilde{a}_{n}, \tilde{a}_{n}\right\rangle\right)=\max _{j}\left(\tilde{a}_{j}\right) .
$$

2) If $w=(0,0, \ldots, 1)^{T}$, then the IITFOWA operator is reduced to the minimum operator:

$$
\operatorname{IITFOWA}_{w}\left(\left\langle\tilde{a}_{1}, \tilde{a}_{1}\right\rangle,\left\langle\tilde{a}_{2}, \tilde{a}_{2}\right\rangle, \ldots,\left\langle\tilde{a}_{n}, \tilde{a}_{n}\right\rangle\right)=\min _{j}\left(\tilde{a}_{j}\right) \text {. }
$$

\section{The definitions of similarity degree and the SD-IITFOWA operator}

\subsection{The definition of similarity degree}

Definition 5. Let $A_{1}=\left\langle\left(a_{1}^{1}, a_{2}^{1}, a_{3}^{1}, a_{4}^{1}\right),\left(b_{1}^{1}, b_{2}^{1}, b_{3}^{1}, b_{4}^{1}\right)\right\rangle$ and $A_{2}=\left\langle\left(a_{1}^{2}, a_{2}^{2}, a_{3}^{2}, a_{4}^{2}\right),\left(b_{1}^{2}, b_{2}^{2}, b_{3}^{2}, b_{4}^{2}\right)\right\rangle$ be two intuitionistic trapezoidal fuzzy numbers, then, we define the weighted distance between $A_{1}$ and $A_{2}$ as follows:

$$
\begin{array}{r}
d\left(A_{1}, A_{2}\right)=\frac{1}{8}\left(\left|a_{1}^{1}-a_{1}^{2}\right|+\left|a_{2}^{1}-a_{2}^{2}\right|+\left|a_{3}^{1}-a_{3}^{2}\right|+\left|a_{4}^{1}-a_{4}^{2}\right|+\right. \\
\left.\left|b_{1}^{1}-b_{1}^{2}\right|+\left|b_{2}^{1}-b_{2}^{2}\right|+\left|b_{3}^{1}-b_{3}^{2}\right|+\left|b_{4}^{1}-b_{4}^{2}\right|\right) .
\end{array}
$$

$\mathrm{Xu}$ and Yager (2009) defined the degree of similarity between two intuitionistic fuzzy numbers for consensus analysis in fuzzy group decision making. In the following, we shall extend the idea of this similarity to develop a new similarity measure between two intuitionistic trapezoidal fuzzy numbers. 
Definition 6. Let $A_{1}=\left\langle\left(a_{1}^{1}, a_{2}^{1}, a_{3}^{1}, a_{4}^{1}\right),\left(b_{1}^{1}, b_{2}^{1}, b_{3}^{1}, b_{4}^{1}\right)\right\rangle$ and $A_{2}=\left\langle\left(a_{1}^{2}, a_{2}^{2}, a_{3}^{2}, a_{4}^{2}\right),\left(b_{1}^{2}, b_{2}^{2}, b_{3}^{2}, b_{4}^{2}\right)\right\rangle$ be two intuitionistic trapezoidal fuzzy numbers, and $\bar{A}_{2}=\left\langle\left(b_{1}^{2}, b_{2}^{2}, b_{3}^{2}, b_{4}^{2}\right),\left(a_{1}^{2}, a_{2}^{2}, a_{3}^{2}, a_{4}^{2}\right)\right\rangle$ be the complement of $A_{2}$, then:

$$
\vartheta\left(A_{1}, A_{2}\right)= \begin{cases}1 & \text { if } A_{1}=A_{2}=\bar{A}_{2} ; \\ \frac{d\left(A_{1}, \bar{A}_{2}\right)}{d\left(A_{1}, A_{2}\right)+d\left(A_{1}, \bar{A}_{2}\right)}, & \text { otherwise }\end{cases}
$$

is called the degree of similarity between $A_{1}$ and $A_{2}$.

According to Eq. (7), we know that $\vartheta\left(A_{1}, A_{2}\right)$ have the following desirable properties:

(1) $0 \leq \vartheta\left(A_{1}, A_{2}\right) \leq 1$;

(2) $\vartheta\left(A_{1}, A_{2}\right)=\vartheta\left(A_{2}, A_{1}\right)=\vartheta\left(\bar{A}_{1}, \bar{A}_{2}\right)$;

(3) $\vartheta\left(A_{1}, \bar{A}_{2}\right)=\vartheta\left(\bar{A}_{1}, A_{2}\right)$;

(4) $\vartheta\left(A_{1}, A_{2}\right)=1 \Leftrightarrow A_{1}=A_{2}$ which means the identity of $A_{1}$ and $A_{2}$;

(5) $\vartheta\left(A_{1}, A_{2}\right)>0.5 \Leftrightarrow d\left(A_{1}, A_{2}\right)<d\left(A_{1}, \bar{A}_{2}\right)$ which means that $A_{1}$ is more similar to $A_{2}$ than $\bar{A}_{2}$;

(6) $\vartheta\left(A_{1}, A_{2}\right)=0.5 \Leftrightarrow d\left(A_{1}, A_{2}\right)=d\left(A_{1}, \bar{A}_{2}\right) \neq 0$ which means that $A_{1}$ is the same extent similar to $A_{2}$ and $\bar{A}_{2}$;

(7) $\vartheta\left(A_{1}, A_{2}\right)<0.5 \Leftrightarrow d\left(A_{1}, A_{2}\right)>d\left(A_{1}, \bar{A}_{2}\right)$ which means that $A_{1}$ is more similar to $\bar{A}_{2}$ than $A_{2}$;

(8) $\vartheta\left(A_{1}, A_{2}\right)=0 \Leftrightarrow A_{1}=\bar{A}_{2}$ which means the complete dissimilarity of $A_{1}$ and $A_{2}$.

In the following, we shall apply the developed similarity measure between two intuitionistic trapezoidal fuzzy numbers to group decision making based on intuitionistic trapezoidal fuzzy decision matrix.

Definition 7. Let $\tilde{R}=\left(\tilde{r}_{i j}\right)_{m \times n}$ be a $m \times n$ matrix. If all $\tilde{r}_{i j}$ are ITFNs, and $\tilde{r}_{i j}=\left\langle\left(a_{i j}, b_{i j}, c_{i j}, d_{i j}\right),\left(e_{i j}, f_{i j}, g_{i j}, h_{i j}\right)\right\rangle, d_{i j}+h_{i j} \leq 1, i=1, \ldots, m ; j=1, \ldots, n$, then we call $\tilde{R}$ an intuitionistic trapezoidal fuzzy decision matrix.

Definition 8. Let $\tilde{R}^{(k)}=\left(\tilde{r}_{i j}^{(k)}\right)_{m \times n}(k=1,2, \ldots, t)$ be $t$ intuitionistic trapezoidal fuzzy decision matrices given by experts $e_{k}(k=1,2, \ldots, m)$ respectively, then the aggregated intuitionistic trapezoidal fuzzy decision matrix $\bar{R}=\left(\bar{r}_{i j}\right)_{m \times n}$ is defined as following:

$$
\bar{r}_{i j}=\left\langle\left(\bar{a}_{i j}, \bar{b}_{i j}, \bar{c}_{i j}, \bar{d}_{i j}\right),\left(\bar{e}_{i j}, \bar{f}_{i j}, \bar{g}_{i j}, \bar{h}_{i j}\right)\right\rangle, \bar{d}_{i j}+\bar{h}_{i j} \leq 1, i=1, \ldots, m ; j=1, \ldots, n,
$$

where $\bar{r}_{i j}=\sum_{k=1}^{t} \frac{1}{t} \tilde{r}_{i j}^{(k)}$.

Definition 9. Let $\tilde{R}^{(k)}=\left(\tilde{r}_{i j}^{(k)}\right)_{m \times n}(k=1,2, \ldots, t)$ be $t$ intuitionistic trapezoidal fuzzy decision matrices, and $\bar{R}=\left(\bar{r}_{i j}\right)_{m \times n}$ be their aggregated intuitionistic trapezoidal fuzzy decision matrix, then:

$$
\dot{\vartheta}\left(\tilde{R}^{(k)}, \bar{R}\right)=\frac{1}{m \times n} \sum_{i=1}^{m} \sum_{j=1}^{n} \vartheta\left(\tilde{r}_{i j}^{(k)}, \bar{r}_{i j}\right),
$$


is called the degree of similarity between $\tilde{R}^{(k)}$ and $\bar{R}$, where $\vartheta\left(\tilde{r}_{i j}^{(k)}, \bar{r}_{i j}\right)$ is the similarity between $\tilde{r}_{i j}^{(k)}$ and $\bar{r}_{i j}$, calculated by Eq. (7).

The greater the value of $\dot{\vartheta}\left(\tilde{R}^{(k)}, \bar{R}\right)$, the greater the similarity degree (SD) between intuitionistic trapezoidal fuzzy decision matrixes $\tilde{R}^{(k)}$ and $\bar{R}$. By Definition 9 , we can obtain the following theorems:

Theorem 7. Let $\tilde{R}^{(k)}=\left(\tilde{r}_{i j}^{(k)}\right)_{m \times n}(k=1,2, \ldots, t)$ be $t$ intuitionistic trapezoidal fuzzy decision matrixes, $\bar{R}=\left(\bar{r}_{i j}\right)_{n \times n}$ be their aggregated decision matrix, then:

(1) $0 \leq \dot{\vartheta}\left(\tilde{R}^{(k)}, \bar{R}\right) \leq 1$,

(2) $\dot{\vartheta}\left(\tilde{R}^{(k)}, \bar{R}\right)=1$ if and only if $\tilde{R}^{(k)}$ and $\tilde{R}$ are identical.

\section{Proof:}

(1) By Definition 9, we have:

$$
0 \leq \vartheta\left(\tilde{r}_{i j}^{(k)}, \bar{r}_{i j}\right) \leq 1, i=1, \ldots, m ; j=1, \ldots, n,
$$

then:

$$
0 \leq \dot{\vartheta}\left(\tilde{R}^{(k)}, \bar{R}\right)=\frac{1}{m \times n} \sum_{i=1}^{m} \sum_{j=1}^{n} \vartheta\left(\tilde{r}_{i j}^{(k)}, \bar{r}_{i j}\right) \leq 1 .
$$

(2) Necessity. If $\dot{\vartheta}\left(\tilde{R}^{(k)}, \bar{R}\right)=1$, then $\vartheta\left(\tilde{r}_{i j}^{(k)}, \bar{r}_{i j}\right)=1$, for all $i, j \in N$, i.e., $\tilde{r}_{i j}^{(k)}=\bar{r}_{i j}$, for all $i, j \in N$. Therefore, $\tilde{R}^{(k)}$ and $\bar{R}$ are identical.

(3) Sufficiency. If $\tilde{R}^{(k)}$ and $\bar{R}$ are identical, then $\tilde{r}_{i j}^{(k)}=\bar{r}_{i j}$, for all $i, j \in N$, i.e., $\vartheta\left(\tilde{r}_{i j}^{(k)}, \bar{r}_{i j}\right)=1$, for all $i, j \in N$. Therefore, $\dot{\vartheta}\left(\tilde{R}^{(k)}, \bar{R}\right)=1$.

Theorem 8. Let $\tilde{R}^{(k)}=\left(\tilde{r}_{i j}^{(k)}\right)_{m \times n}(k=1,2, \ldots, t)$ be $t$ intuitionistic trapezoidal fuzzy decision matrixes and $\tilde{R}=\left(\tilde{r}_{i j}\right)_{n \times n}$ be their aggregated decision matrix, then, we have:

(1) $\dot{\vartheta}\left(\tilde{R}^{(k)}, \tilde{R}^{(k)}\right)=1$,

(2) $\dot{\vartheta}\left(\tilde{R}^{(k)}, \tilde{R}^{(l)}\right)=\dot{\vartheta}\left(\tilde{R}^{(l)}, \tilde{R}^{(k)}\right)$,

(3) If $\dot{\vartheta}\left(\tilde{R}^{(k)}, \bar{R}\right)=1$ and $\dot{\vartheta}\left(\tilde{R}^{(l)}, \bar{R}\right)=1$, then $\dot{\vartheta}\left(\tilde{R}^{(k)}, \tilde{R}^{(l)}\right)=1$.

\section{Proof:}

(1) Since $\vartheta\left(\tilde{r}_{i j}^{(k)}, \tilde{r}_{i j}^{(k)}\right)=1$, for all $i, j \in N$, then:

$$
\dot{\vartheta}\left(\tilde{R}^{(k)}, \bar{R}\right)=\frac{1}{m \times n} \sum_{i=1}^{m} \sum_{j=1}^{n} \vartheta\left(\tilde{r}_{i j}^{(k)}, \bar{r}_{i j}\right)=1 .
$$

(2) Since $\vartheta\left(\tilde{r}_{i j}^{(k)}, \bar{r}_{i j}\right)=\vartheta\left(\bar{r}_{i j}, \tilde{r}_{i j}^{(k)}\right)$, for all $i, j \in N$, then:

$$
\dot{\vartheta}\left(\tilde{R}^{(k)}, \bar{R}\right)=\frac{1}{m \times n} \sum_{i=1}^{m} \sum_{j=1}^{n} \vartheta\left(\tilde{r}_{i j}^{(k)}, \bar{r}_{i j}\right)=\frac{1}{m \times n} \sum_{i=1}^{m} \sum_{j=1}^{n} \vartheta\left(\bar{r}, \tilde{r}_{i j}^{(k)}\right)=\dot{\vartheta}\left(\bar{R}, \tilde{R}^{(k)}\right) .
$$


(3) If $\dot{\vartheta}\left(\tilde{R}^{(k)}, \bar{R}\right)=1$ and $\dot{\vartheta}\left(\tilde{R}^{(l)}, \bar{R}\right)=1$, then $\tilde{r}_{i j}^{(k)}=\bar{r}_{i j}$, for all $i, j \in N, \tilde{r}_{i j}^{(l)}=\bar{r}_{i j}$, for all $i, j \in N$, thus $\tilde{r}_{i j}^{(k)}=\tilde{r}_{i j}^{(l)}$, for all $i, j \in N$. Therefore, $\dot{\vartheta}\left(\tilde{R}^{(k)}, \tilde{R}^{(l)}\right)=1$.

\subsection{The SD-IITFOWA operator}

In some GDM problems, we usually have not the information about DM weights. However, in this situation, each DM always can have an associated similarity degree (SD) value. Thus, the more similarity is the information provided by the DM, the higher the weighting value should be placed on that information. In this section, we define the concept of the SD-IITFOWA operator and present a method to determine its associated weights.

Definition 10. If a set of DMs $D=\left\{d_{1}, d_{2}, \ldots, d_{t}\right\}$ provides preference about a set of alternatives $A=\left\{A_{1}, A_{2}, \ldots, A_{m}\right\}$ by means of intuitionistic trapezoidal fuzzy decision matrix $\tilde{R}^{(k)}=\left(\tilde{r}_{i j}^{(k)}\right)_{m \times n}(k=1,2, \ldots, t)$, then a SD-IITFOWA operator is an IITFOWA operator in which its order-inducing values is the set of SD values $\left\{\dot{\vartheta}\left(\tilde{R}^{(1)}, \bar{R}\right), \ldots, \dot{\vartheta}\left(\tilde{R}^{(k)}, \bar{R}\right), \ldots, \dot{\vartheta}\left(\tilde{R}^{(t)}, \bar{R}\right)\right\}$.

Before implementing this operator to aggregate individual intuitionistic trapezoidal fuzzy decision matrix, we calculate its associated weights of $\tilde{R}^{(k)}$ by using the function of its SD value considering the following three cases:

(1) If intuitionistic trapezoidal fuzzy decision matrixes $\tilde{R}^{(k)}=\left(\tilde{r}_{i j}^{(k)}\right)_{m \times n}(k=1,2, \ldots, t)$ are all identical, then let

$$
w^{(k)}=\frac{1}{t}
$$

(2) If intuitionistic trapezoidal fuzzy decision matrixes $\tilde{R}^{(k)}=\left(\tilde{r}_{i j}^{(k)}\right)_{m \times n}(k=1,2, \ldots, t)$ are non-identical, then:

$$
w^{(k)}=\frac{\left(\dot{\vartheta}\left(\tilde{R}^{(k)}, \bar{R}\right)\right)^{\alpha}}{\sum_{k=1}^{t}\left(\dot{\vartheta}\left(\tilde{R}^{(k)}, \bar{R}\right)\right)^{\alpha}},
$$

where, $\alpha>0$.

(3) Suppose that there exist $l$ identical intuitionistic trapezoidal fuzzy decision matrixes and $t-l$ non-identical intuitionistic trapezoidal fuzzy decision matrixes. Without loss generality, we assume that $A^{(1)}, A^{(2)}, \ldots, A^{(l)}(1 \leq l \leq t)$ are identical while $A^{(l+1)}, A^{(l+2)}, \ldots, A^{(t)}$ are non-identical, and then have:

$$
w^{(k)}=\left\{\begin{array}{ll}
\frac{1}{\left[l+\sum_{l=k+1}^{m}\left(\dot{\vartheta}\left(\tilde{R}^{(k)}, \bar{R}\right)\right)^{\alpha}\right]}, & 1 \leq k \leq l \\
\frac{\left(\dot{\vartheta}\left(\tilde{R}^{(k)}, \bar{R}\right)\right)^{\alpha}}{\left[l+\sum_{l=k+1}^{m}\left(\dot{\vartheta}\left(\tilde{R}^{(k)}, \bar{R}\right)\right)^{\alpha}\right]}, & l+1 \leq k \leq m
\end{array},\right.
$$

where, $w^{(k)}$ is the weighting value of intuitionistic trapezoidal fuzzy decision matrix $\tilde{R}^{(k)}=\left(\tilde{r}_{i j}^{(k)}\right)_{m \times n}(k=1,2, \ldots, t), \alpha>0$. 
Definition 11. If $\tilde{R}^{(k)}=\left(\tilde{r}_{i j}^{(k)}\right)_{m \times n}(k=1,2, \ldots, t)$ are the intuitionistic trapezoidal fuzzy decision matrixes provided by $t$ DMs, then the collective decision matrix (CDM) $\widehat{R}=\left(\widehat{r}_{i j}\right)_{n \times n}$ by SD-IITOWA operator is expressed as follows:

$$
\begin{gathered}
\left.\widehat{R}=\operatorname{SD} \text {-IITFOWA }\left(\left\langle\dot{\vartheta}\left(\tilde{R}^{(1)}, \bar{R}\right), \tilde{R}^{(1)}\right\rangle,\left\langle\dot{\vartheta}\left(\tilde{R}^{(2)}, \bar{R}\right), \tilde{R}^{(2)}\right\rangle, \cdots,\left\langle\dot{\vartheta}\left(\tilde{R}^{(t)}, \bar{R}\right), \bar{R}\right), \tilde{R}^{(t)}\right\rangle\right)= \\
\operatorname{SD-IITFOWA}\left(\left\langle\dot{\vartheta}\left(\tilde{R}^{(\sigma(1))}, \bar{R}\right), \tilde{R}^{(\sigma(1))}\right\rangle,\left\langle\dot{\vartheta}\left(\tilde{R}^{(\sigma(2))}, \bar{R}\right), \tilde{R}^{(\sigma(2))}\right\rangle, \cdots,\left\langle\dot{\vartheta}\left(\tilde{R}^{(\sigma(t))}, \bar{R}\right), \tilde{R}^{(\sigma(t))}\right\rangle\right)= \\
\gamma_{\sigma(1)} \tilde{R}^{(\sigma(1))} \oplus \gamma_{\sigma(2)} \tilde{R}^{(\sigma(2))} \oplus \ldots \oplus \gamma_{\sigma(t)} \tilde{R}^{(\sigma(t))} ; \\
\widehat{r}_{i j}=\gamma_{\sigma(1)} \tilde{r}_{i j}^{(\sigma(1))} \oplus \gamma_{\sigma(2)} \tilde{r}_{i j}^{(\sigma(2))} \oplus \ldots \oplus \gamma_{\sigma(t)} \tilde{r}_{i j}^{(\sigma(t))}= \\
\left(1-\prod_{k=1}^{t}\left(1-u_{\sigma(k)}\right)^{\gamma_{\sigma(k)}}, \prod_{j=1}^{n}\left(u_{\sigma(j)}\right)^{\gamma_{\sigma(k)}}\right),
\end{gathered}
$$

where $\left(\sigma(1), \sigma(2), \ldots, \sigma(n)\right.$ is a permutation of $(1,2, \ldots, n)$ such that $\dot{\vartheta}\left(\tilde{R}^{(\sigma(n-1))}, \bar{R}\right) \geq \dot{\vartheta}\left(\tilde{R}^{(\sigma(n))}, \bar{R}\right)$ and $\gamma_{\sigma(l-1)} \geq \gamma_{\sigma(l)}$ for all $l=2, \ldots, m ;\left\langle\dot{\vartheta}\left(\tilde{R}^{(\sigma(k))}, \bar{R}\right), \tilde{R}^{(\sigma(k))}\right\rangle$ is the two tuple with $\dot{\vartheta}\left(\tilde{R}^{(\sigma(k))}, \bar{R}\right)$ the $l$-th biggest value in the set $\left\{\dot{\vartheta}\left(\tilde{R}^{(\sigma(1))}, \bar{R}\right), \dot{\vartheta}\left(\tilde{R}^{(\sigma(2))}, \bar{R}\right), \ldots, \dot{\vartheta}\left(\tilde{R}^{(\sigma(t))}, \bar{R}\right)\right\}$; $\gamma=\left(\gamma_{\sigma(1)}, \gamma_{\sigma(2)}, \ldots, \gamma_{\sigma(m)}\right)^{T}$ is a weighting vector, such that $\sum_{l=1}^{m} \gamma_{\sigma(l)}=1, \gamma_{\sigma(l)} \in[0,1]$.

\section{A SD-IITFOWA operator and TOPSIS method based approach to MAGDM problems}

Let $A=\left\{A_{1}, A_{2}, \ldots, A_{m}\right\}$ be a discrete set of alternatives, and $U=\left\{u_{1}, u_{2}, \ldots, u_{n}\right\}$ be the set of attributes, $W=\left\{w_{1}, w_{2}, \ldots, w_{n}\right\}$ is the weighting vector of the attribute $w_{j}(j=1,2, \ldots, n)$, where $w_{j}>0, \sum_{j=1}^{n} w_{j}=1$. Let $D=\left\{d_{1}, d_{2}, \ldots, d_{t}\right\}$ be the set of decision makers. Suppose that $\tilde{R}^{(k)}=\left(\tilde{r}_{i j}^{(k)}\right)_{m \times n}(k=1,2, \ldots, t)$ are the intuitionistic trapezoidal fuzzy decision matrixes.

In the following, we shall develop an SD-IITFOWA operator and TOPSIS method based approach to resolve multiple attribute group decision making with intuitionistic trapezoidal fuzzy information, which involves the following steps:

Step 1. Utilize the arithmetic averaging operator aggregate all the decision matrices $\tilde{R}^{(k)}$ $(k=1,2, \ldots, t)$ into an aggregated decision matrix $\bar{R}=\left(\bar{r}_{i j}\right)_{n \times n}$, where:

$$
\bar{r}_{i j}=\frac{1}{t} \sum_{k=1}^{t} \tilde{r}_{i j}^{(k)}, i=1, \ldots, m ; j=1, \ldots, n \text {. }
$$

Step 2. By Eq. (8), we calculate the similarity degree (SD) $\dot{\vartheta}\left(\tilde{R}^{(k)}, \tilde{R}\right)(k=1,2, \ldots, t)$.

Step 3. Based on the similarity degree $\dot{\vartheta}\left(\tilde{R}^{(k)}, \tilde{R}\right)$, we can determine the weights of the experts $W=\left\{w^{(2)}, \ldots, w^{(k)}, \ldots, w^{(t)}\right\}$, where $w^{(k)}=\frac{\left(\dot{\vartheta}\left(\tilde{R}^{(k)}, \bar{R}\right)\right)^{\alpha}}{\sum_{k=1}^{t}\left(\dot{\vartheta}\left(\tilde{R}^{(k)}, \bar{R}\right)\right)^{\alpha}}$. 
Step 4. If $\dot{\vartheta}\left(\tilde{R}^{(k)}, \tilde{R}\right) \geq \dot{\vartheta}\left(\tilde{R}^{(l)}, \tilde{R}\right)$, then the $k$-th expert has more similarity than the $l$-th expert and we have $w^{(k)} \geq w^{(l)}$. Thus, the more importance is placed on the most similarity one By the SD-IITFOWA operator, we aggregate all the individual decision opinions into a group opinion and construct the collective decision matrix $(\mathrm{CDM}) \widehat{R}=\left(\widehat{r}_{i j}\right)_{n \times n}$, where $\widehat{r}_{i j}$ is calculated by Eq. (13).

Step 5. Let $J_{1}$ be the set of benefit attributes and $J_{2}$ be the set of cost attributes, respectively. $A^{*}$ is intuitionistic fuzzy positive-ideal solution and $A^{-}$is intuitionistic trapezoidal fuzzy negative-ideal solution. Then $A^{*}$ and $A^{-}$are defined as follows:

$$
\begin{aligned}
& A^{*}=\left[\left\langle r_{j}^{*}=(1,1,1,1),(0,0,0,0)\right\rangle\left|j \in J_{1},\left\langle r_{j}^{*}=(0,0,0,0),(1,1,1,1)\right\rangle\right| j \in J_{2}\right] ; \\
& A^{-}=\left[\left\langle r_{j}^{-}=(0,0,0,0),(1,1,1,1)\right\rangle\left|j \in J_{1},\left\langle r_{j}^{-}=(1,1,1,1),(0,0,0,0)\right\rangle\right| j \in J_{2}\right] .
\end{aligned}
$$

Step 6. By Eq. (6), we calculate the separation measures, $S_{i}^{-}$and $S_{i}^{\star}$, of each alternative from intuitionistic trapezoidal fuzzy positive-ideal and negative-ideal solutions as follows:

$$
\begin{aligned}
& S_{i}^{*}=\frac{1}{n} \sum_{j=1}^{n} w_{j} d\left(r_{i j}^{\prime}, r_{j}^{*}\right) ; \\
& S_{i}^{-}=\frac{1}{n} \sum_{j=1}^{n} w_{j} d\left(r_{i j}^{\prime}, r_{j}^{-}\right) .
\end{aligned}
$$

Step 7. Calculate the relative closeness coefficient to the intuitionistic ideal solution. The relative closeness coefficient of an alternative $A_{i}$ with respect to the intuitionistic trapezoidal fuzzy positive-ideal solution $A^{*}$ is defined as follows:

$$
C_{i}=\frac{S_{i}^{-}}{S_{i}^{-}+S_{i}^{*}}, \text { where } 0 \leq C_{i} \leq 1 \text {. }
$$

Step 8. After the relative closeness coefficient of each alternative is determined, alternatives are ranked according to the descending order of $C_{i}$.

\section{A numerical example}

A computer company is desirable to select the most appropriate supplier for one of the key elements in its manufacturing process. After pre-evaluation, three suppliers have remained as alternatives for further evaluation. Five criteria are considered as: $u_{1}$ - Product quality; $u_{2}$ - Service performance; $u_{3}$ - Price; $u_{4}$ - Environment management; $u_{5}$ - Delivery date (whose weighting vector $\left.W=(0.25,0.20,0.15,0.10,0.30)^{T}\right)$. This company has a group of DMs form four consultancy departments: $d_{1}$ is from the financial department; $d_{2}$ is from the purchasing department; $d_{3}$ is from the quality inspection department; $d_{4}$ is from the engineering department.

Procedure for the selection of supplier contains the following steps:

Step 1. Construct the aggregated intuitionistic trapezoidal fuzzy decision matrix based on the opinions of decision makers. The linguistic terms shown in Table 1 are used to rate each alternative supplier with respect to each criterion by decision maker.

The assessments given by these DMs to five alternatives were shown in Table 2, Table 3, Table 4, and Table 5. 
Table 1. Linguistic terms for rating the alternatives

Linguistic terms

\begin{tabular}{|c|c|c|c|c|c|}
\hline \multicolumn{4}{|c|}{ Very good (VG)/very high (VH) } & \multicolumn{2}{|c|}{$[(1.0,1.0,1.0,1.0),(0.0$} \\
\hline \multicolumn{4}{|c|}{ Good (G)/high (H) } & \multicolumn{2}{|c|}{$[(0.7,0.8,0.9,1.0),(0.0$} \\
\hline \multicolumn{4}{|c|}{ Medium good (MG)/medium high $(\mathrm{MH})$} & \multicolumn{2}{|c|}{$[(0.5,0.6,0.7,0.8),(0.1$} \\
\hline \multicolumn{4}{|c|}{ Fair $(\mathrm{F}) /$ medium $(\mathrm{M})$} & \multicolumn{2}{|c|}{$[(0.3,0.4,0.5,0.6),(0.2$} \\
\hline \multicolumn{4}{|c|}{ Medium bad (MB)/medium low (ML) } & \multicolumn{2}{|c|}{$[(0.1,0.2,0.3,0.4),(0.5$} \\
\hline \multicolumn{4}{|c|}{$\operatorname{Bad}(\mathrm{B}) / \mathrm{low}(\mathrm{L})$} & \multicolumn{2}{|c|}{$[(0.0,0.1,0.2,0.3),(0.7$} \\
\hline \multicolumn{4}{|c|}{ Very bad (VB)/very low (VL) } & \multicolumn{2}{|c|}{$[(0.0,0.0,0.0,0.0),(1.0$} \\
\hline \multirow{2}{*}{ Suppliers } & \multicolumn{5}{|c|}{ Criteria } \\
\hline & $u_{1}$ & $u_{2}$ & $u_{3}$ & $u_{4}$ & $u_{5}$ \\
\hline$A_{1}$ & ML & $\mathrm{VH}$ & VH & M & ML \\
\hline$A_{2}$ & $\mathrm{H}$ & $\mathrm{MH}$ & ML & $\mathrm{VH}$ & $\mathrm{L}$ \\
\hline$A_{3}$ & $\mathrm{MH}$ & M & VH & VL & $\mathrm{MH}$ \\
\hline
\end{tabular}

Table 3. Assessments of the four supplies by the DM $d_{2}$ based on each criterion

\begin{tabular}{cccccc}
\hline \multirow{2}{*}{ Suppliers } & \multicolumn{5}{c}{ Criteria } \\
\cline { 2 - 6 } & $u_{1}$ & $u_{2}$ & $u_{3}$ & $u_{4}$ & $u_{5}$ \\
\hline$A_{1}$ & $\mathrm{MH}$ & $\mathrm{L}$ & $\mathrm{H}$ & $\mathrm{VH}$ & $\mathrm{VH}$ \\
$A_{2}$ & $\mathrm{H}$ & $\mathrm{MH}$ & $\mathrm{M}$ & $\mathrm{L}$ & $\mathrm{H}$ \\
$A_{3}$ & $\mathrm{M}$ & $\mathrm{H}$ & $\mathrm{MH}$ & $\mathrm{M}$ & $\mathrm{L}$ \\
\hline
\end{tabular}

Table 4 . Assessments of the four supplies by the $\mathrm{DM} d_{3}$ based on each criterion

\begin{tabular}{cccccc}
\hline \multirow{2}{*}{ Suppliers } & \multicolumn{5}{c}{ Criteria } \\
\cline { 2 - 6 } & $u_{1}$ & $u_{2}$ & $u_{3}$ & $u_{4}$ & $u_{5}$ \\
\hline$A_{1}$ & $\mathrm{ML}$ & $\mathrm{MH}$ & $\mathrm{H}$ & $\mathrm{VH}$ & $\mathrm{H}$ \\
$A_{2}$ & $\mathrm{VH}$ & $\mathrm{M}$ & $\mathrm{VH}$ & $\mathrm{M}$ & $\mathrm{ML}$ \\
$A_{3}$ & $\mathrm{VH}$ & $\mathrm{H}$ & $\mathrm{H}$ & $\mathrm{ML}$ & $\mathrm{MH}$ \\
\hline
\end{tabular}

Table 5. Assessments of the four supplies by the $\mathrm{DM} d_{4}$ based on each criterion

\begin{tabular}{cccccc}
\hline \multirow{2}{*}{ Suppliers } & \multicolumn{5}{c}{ Criteria } \\
\cline { 2 - 6 } & $u_{1}$ & $u_{2}$ & $u_{3}$ & $u_{4}$ & $u_{5}$ \\
\hline$A_{1}$ & $\mathrm{ML}$ & $\mathrm{M}$ & $\mathrm{M}$ & $\mathrm{MH}$ & $\mathrm{L}$ \\
$A_{2}$ & $\mathrm{~L}$ & $\mathrm{ML}$ & $\mathrm{ML}$ & $\mathrm{L}$ & $\mathrm{MH}$ \\
$A_{3}$ & $\mathrm{~L}$ & $\mathrm{M}$ & $\mathrm{L}$ & $\mathrm{M}$ & $\mathrm{ML}$ \\
\hline
\end{tabular}


The intuitionistic trapezoidal fuzzy decision matrices based on decision maker' opinions are constructed as follows:

$R^{(1)}=$

$\left(\begin{array}{ccc}A_{1} & A_{2} & A_{3} \\ u_{1}\langle(0.1,0.2,0.3,0.4),(0.5,0.6,0.7,0.8)\rangle & \langle(0.7,0.8,0.9,1.0),(0.0,0.1,0.2,0.3)\rangle & \langle(0.5,0.6,0.7,0.8),(0.1,0.2,0.3,0.4) \\ u_{2}\langle(1.0,1.0,1.0,1.0),(0.0,0.0,0.0,0.0)\rangle & \langle(0.5,0.6,0.7,0.8),(0.1,0.2,0.3,0.4)\rangle & \langle(0.3,0.4,0.5,0.6),(0.2,0.3,0.4,0.5)\rangle \\ u_{3}\langle(1.0,1.0,1.0,1.0),(0.0,0.0,0.0,0.0)\rangle & \langle(0.1,0.2,0.3,0.4),(0.5,0.6,0.7,0.8)\rangle & \langle(1.0,1.0,1.0,1.0),(0.0,0.0,0.0,0.0)\rangle \\ u_{4}\langle(0.3,0.4,0.5,0.6),(0.2,0.3,0.4,0.5)\rangle & \langle(1.0,1.0,1.0,1.0),(0.0,0.0,0.0,0.0)\rangle & \langle(0.0,0.0,0.0,0.0),(1.0,1.0,1.0,1.0)\rangle \\ u_{5}\langle(0.1,0.2,0.3,0.4),(0.5,0.6,0.7,0.8)\rangle & \langle(0.0,0.1,0.2,0.3),(0.7,0.8,0.9,1.0)\rangle & \langle(0.5,0.6,0.7,0.8),(0.1,0.2,0.3,0.4)\end{array}\right) ;$

$R^{(2)}=$

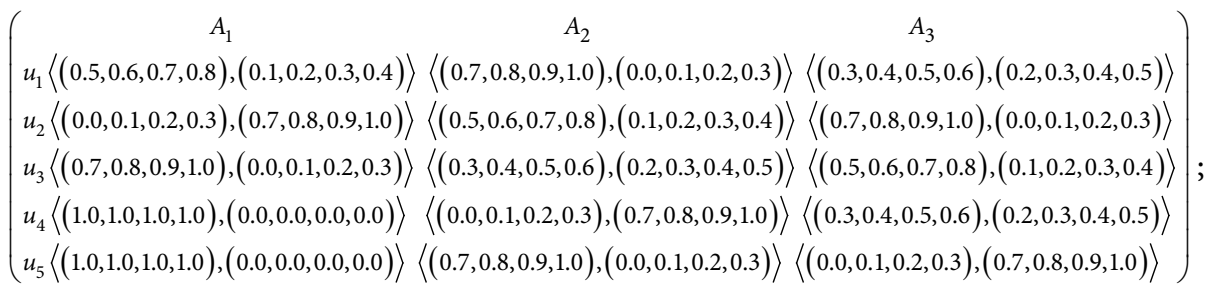

$R^{(3)}=$

$\left(\begin{array}{ccc}A_{1} & A_{2} & A_{3} \\ u_{1}\langle(0.1,0.2,0.3,0.4),(0.5,0.6,0.7,0.8)\rangle & \langle(1.0,1.0,1.0,1.0),(0.0,0.0,0.0,0.0)\rangle & \langle(1.0,1.0,1.0,1.0),(0.0,0.0,0.0,0.0)\rangle \\ u_{2}\langle(0.5,0.6,0.7,0.8),(0.1,0.2,0.3,0.4)\rangle & \langle(0.3,0.4,0.5,0.6),(0.2,0.3,0.4,0.5)\rangle & \langle(0.7,0.8,0.9,1.0),(0.0,0.1,0.2,0.3)\rangle \\ u_{3}\langle(0.7,0.8,0.9,1.0),(0.0,0.1,0.2,0.3)\rangle & \langle(1.0,1.0,1.0,1.0),(0.0,0.0,0.0,0.0)\rangle\langle(0.7,0.8,0.9,1.0),(0.0,0.1,0.2,0.3)\rangle \\ u_{4}\langle(1.0,1.0,1.0,1.0),(0.0,0.0,0.0,0.0)\rangle & \langle(0.3,0.4,0.5,0.6),(0.2,0.3,0.4,0.5)\rangle & \langle(0.1,0.2,0.3,0.4),(0.5,0.6,0.7,0.8)\rangle \\ u_{5}\langle(0.7,0.8,0.9,1.0),(0.0,0.1,0.2,0.3)\rangle & \langle(0.1,0.2,0.3,0.4),(0.5,0.6,0.7,0.8)\rangle & \langle(0.5,0.6,0.7,0.8),(0.1,0.2,0.3,0.4)\end{array}\right) ;$

$R^{(4)}=$

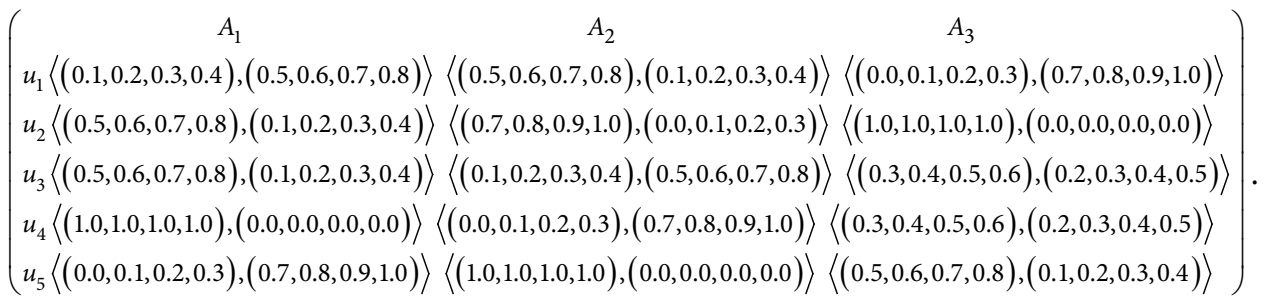

Utilize Eq. (14), all the decision matrices $\tilde{R}^{(k)}(k=1,2,3,4)$ are synthesized into a aggregated decision matrix $\bar{R}=\left(\bar{r}_{i j}\right)_{n \times n}$ :

$\bar{R}=$

$\left(\begin{array}{cc}A_{1} & A_{2} \\ u_{1}\langle(0.1,0.2,0.3,0.4),(0.4,0.5,0.6,0.7)\rangle\langle(0.7,0.7,0.8,0.8),(0.2,0.3,0.3,0.4)\rangle\langle(0.6,0.7,0.7,0.8),(0.3,0.3,0.4,0.5)\rangle \\ u_{2}\langle(0.6,0.7,0.7,0.8),(0.3,0.3,0.4,0.5)\rangle\langle(0.3,0.4,0.5,0.6),(0.2,0.3,0.4,0.5)\rangle\langle(0.5,0.6,0.7,0.8),(0.1,0.2,0.3,0.4)\rangle \\ u_{3}\langle(0.6,0.7,0.7,0.8),(0.3,0.4,0.5,0.5)\rangle\langle(0.4,0.5,0.6,0.7),(0.3,0.4,0.5,0.5)\rangle\langle(0.5,0.6,0.7,0.7),(0.2,0.3,0.4,0.4)\rangle \\ u_{4}\langle(0.7,0.8,0.8,0.9),(0.1,0.1,0.2,0.2)\rangle\langle(0.4,0.5,0.6,0.6),(0.4,0.5,0.6,0.6)\rangle & \langle(0.1,0.20 .30 .4),(0.5,0.6,0.6,0.7)\rangle \\ u_{5}\langle(0.4,0.5,0.6,0.7),(0.3,0.4,0.5,0.5)\rangle\langle(0.2,0.3,0.4,0.5),(0.3,0.4,0.5,0.6)\rangle\langle(0.4,0.50 .60 .7),(0.4,0.5,0.6,0.7)\rangle\end{array}\right)$. 
Step 2. By Eq. (8), we can calculate the similarity degree $\dot{\vartheta}\left(\tilde{R}^{(k)}, \tilde{R}\right)(k=1,2,3,4)$ :

$$
\begin{aligned}
& \dot{\vartheta}\left(\tilde{R}^{(1)}, \bar{R}\right)=0.64 ; \dot{\vartheta}\left(\tilde{R}^{(2)}, \bar{R}\right)=0.67 ; \\
& \dot{\vartheta}\left(\tilde{R}^{(3)}, \bar{R}\right)=0.72 ; \dot{\vartheta}\left(\tilde{R}^{(4)}, \bar{R}\right)=0.45
\end{aligned}
$$

and the intuitionistic trapezoidal fuzzy decision matrices $R^{(1)}, R^{(2)}, R^{(3)}, R^{(4)}$ are re-ordered as follows:

$$
\begin{aligned}
& \tilde{R}^{\sigma(1)}=\tilde{R}^{(3)} ; \tilde{R}^{\sigma(2)}=\tilde{R}^{(2)} ; \\
& \tilde{R}^{\sigma(3)}=\tilde{R}^{(1)} ; \tilde{R}^{\sigma(4)}=\tilde{R}^{(4)} .
\end{aligned}
$$

Using formula (10) with $\alpha=2$, we get the following weights:

$$
\gamma_{\sigma(1)}=0.33 ; \gamma_{\sigma(2)}=0.28 ; \gamma_{\sigma(3)}=0.26 ; \gamma_{\sigma(4)}=0.13
$$

Applying Eq. (12), we can obtain the collective decision matrix (CDM) $\widehat{R}$ as follows: $\widehat{R}=$

$\left(\begin{array}{ccc}A_{1} & A_{2} & A_{3} \\ u_{1}\langle(0.1,0.2,0.3,0.4),(0.4,0.5,0.6,0.7)\rangle & \langle(0.8,0.8,0.9,0.9),(0.1,0.2,0.2,0.3)\rangle & \langle(0.7,0.8,0.8,0.9),(0.2,0.2,0.3,0.4)\rangle \\ u_{2}\langle(0.6,0.7,0.8,0.8),(0.3,0.3,0.4,0.5)\rangle & \langle(0.3,0.4,0.5,0.6),(0.2,0.3,0.4,0.5)\rangle\langle(0.5,0.6,0.7,0.8),(0.1,0.2,0.3,0.4)\rangle \\ u_{3}\langle(0.6,0.7,0.8,0.8),(0.0,0.1,0.2,0.2)\rangle & \langle(0.5,0.6,0.6,0.7),(0.3,0.3,0.4,0.5)\rangle\langle(0.6,0.6,0.7,0.8),(0.1,0.2,0.3,0.3)\rangle \\ u_{4}\langle(0.8,0.8,0.8,0.9),(0.1,0.1,0.1,0.2)\rangle & \langle(0.4,0.5,0.6,0.7),(0.4,0.4,0.5,0.6)\rangle & \langle(0.1,0.2,0.2,0.3),(0.5,0.6,0.7,0.7)\rangle \\ u_{5}\langle(0.5,0.5,0.7,0.8),(0.2,0.3,0.4,0.4)\rangle\langle(0.1,0.2,0.3,0.4),(0.4,0.5,0.6,0.7)\rangle\langle(0.4,0.5,0.6,0.7),(0.3,0.4,0.5,0.6)\rangle\end{array}\right)$.

Step 3. Product quality $u_{1}$, Service performance $u_{2}$ and Environment management $u_{4}$ are benefit criteria $J_{1}=\left\{u_{1}, u_{2}, u_{4}\right\}$. Price $u_{3}$ and Delivery date $u_{5}$ are cost criteria $J_{2}=\left\{u_{3}, u_{5}\right\}$. Then intuitionistic trapezoidal fuzzy positive-ideal solution $A^{\star}$ and intuitionistic trapezoidal fuzzy negative-ideal solution $A^{-}$are obtained as follows:

$$
\begin{aligned}
A^{*}= & {[\langle(1,1,1,1),(0,0,0,0)\rangle,\langle(1,1,1,1),(0,0,0,0)\rangle,\langle(0,0,0,0),(1,1,1,1)\rangle,} \\
& \langle(1,1,1,1),(0,0,0,0)\rangle,\langle(0,0,0,0),(1,1,1,1)\rangle]^{T} ; \\
A^{-}= & {[\langle(0,0,0,0),(1,1,1,1)\rangle,\langle(0,0,0,0),(1,1,1,1)\rangle,\langle(1,1,1,1),(0,0,0,0)\rangle,} \\
& \langle(0,0,0,0),(1,1,1,1)\rangle,\langle(1,1,1,1),(0,0,0,0)\rangle]^{T} .
\end{aligned}
$$

Step 4. The vector of attribute weights is $W=(0.25,0.20,0.15,0.10,0.30)^{T}$. By utilizing Eq. (17), we can calculate the separation measures $S_{i}^{*}$ of each alternative from intuitionistic trapezoidal fuzzy positive-ideal solutions as follows:

$$
S_{1}^{*}=\frac{1}{n} \sum_{j=1}^{n} w_{j} d\left(r_{1 j}^{\prime}, r_{j}^{*}\right)=4.460, S_{2}^{*}=3.015, S_{3}^{*}=3.715,
$$

and by Eq. (18), we have the separation measures $S_{i}^{-}$of each alternative from intuitionistic trapezoidal fuzzy negative-ideal solutions as follows:

$$
S_{1}^{-}=3.540, S_{2}^{-}=4.985, S_{3}^{-}=4.285 \text {. }
$$


According to Eq. (19), we obtain the relative closeness coefficient to the intuitionistic trapezoidal fuzzy ideal solution in Table 6:

$$
C_{1}=\frac{S_{1}^{-}}{S_{1}^{-}+S_{1}^{\star}}=0.4425, C_{2}=0.6231, C_{3}=0.5356 .
$$

Table 6. The relative closeness coefficient of each alternative

\begin{tabular}{cc}
\hline Suppliers & $C_{i}^{-}$ \\
\hline$A_{1}$ & 0.44 \\
$A_{2}$ & 0.62 \\
$A_{3}$ & 0.53 \\
\hline
\end{tabular}

Step 5. Rank all the alternatives $x_{i}(i=1,2,3)$ in accordance with the descending order of $C_{i}^{-}: x_{2} \succ x_{3} \succ x_{1}$, and thus the most desirable alternative is $x_{3}$.

\section{Conclusions}

This paper proposed an approach to MAGDM problems with intuitionistic trapezoidal fuzzy numbers. The concept of similarity degree (SD) for two intuitionistic trapezoidal fuzzy decision matrixes was defined. Then, we presented the SD induced IITFOWA (SD-IITFOWA) operator to aggregate intuitionistic trapezoidal fuzzy numbers. Its novelty is that it aggregates individual opinion in such a way that more importance is placed on the most similarity one. Combining the SD-IITFOWA operator and TOPSIS method, an approach was developed to solve the multiple attribute group decision making (MAGDM) problems in which the attributes are inter-dependent. Finally, some illustrative examples had been given to show the developed method. However, many decision making processes, in the real world, take place in an environment in which the information is not complete. We anticipate that future work will concentrate in the MAGDM problems with intuitionistic trapezoidal fuzzy information in which the decision matrixes are incomplete.

\section{Acknowledgements}

The authors are very grateful to anonymous referees for their valuable comments and suggestions. This work was supported by National Natural Science Foundation of China (NSFC) under the Grant No.71101131, Humanities and Social Science Projects of Chinese Ministry of Education under the Grant No.10YJC630277, Zhejiang Provincial Philosophy and Social Sciences Planning Project of China under the Grant No.13NDJC015Z and Zhejiang Provincial National Science Foundation for Distinguished Young Scholars of China under the Grant No. LR13G010001.

\section{References}

Ashayeri, J.; Tuzkaya, G.; Tuzkaya, U. 2012. Supply chain partners and configuration selection: an intuitionist fuzzy chouquet integral operator based approach, Expert Systems with Applications 39: 3642-3649. http://dx.doi.org/10.1016/j.eswa.2011.09.055 
Atanassov, K. 1986. Intuitionstic fuzzy sets, Fuzzy Sets and Systems 20: 87-96. http://dx.doi.org/10.1016s0165-0114(86)80034-3

Atanassov, K.; Gargov, G. 1989. Interval-valued intuitionistic fuzzy sets, Fuzzy Sets and Systems 31: 343-349. http://dx.doi.org/10.1016/0165-0114(89)90205-4

Boran, F. E.; Genç, S.; Kurt, M.; Akay, D. 2009. A multi-criteria intuitionistic fuzzy group decision making for supplier selection with TOPSIS method, Expert Systems with Applications 36: 11363-11368. http://dx.doi.org/10.1016/j.eswa.2009.03.039

Chiclana, F.; Herrera, F.; Herrera-Viedma, E.; Martínez, L. 2003. A note on the reciprocity in the aggregation of fuzzy preference relations using OWA operators, Fuzzy Sets and Systems 13: 771-783. http://dx.doi.org/10.1016/S0165-0114(02)00433-5

Chiclana, F.; Herrera, F.; Herrera-Viedma, E.; Martínez, L. 2007. Some induced ordered weighted averaging operators and their use for solving group decision-making problems based on fuzzy preference relations, European Journal of Operational Research 182: 383-399.

http://dx.doi.org/10.1016/j.ejor.2006.08.032

Choudhury, A. K.; Shankar, R.; Tiwari, M. K. 2006. Consensus-based intelligent group decision making model for the selection of advanced technology, Decision Support Systems 42: 1776-1799. http://dx.doi.org/10.1016/j.dss.2005.05.001

Devi, K. 2011. Extension of VIKOR method in intuitionistic fuzzy environment for robot selection, Expert Systems with Applications 38: 14163-14168. http://dx.doi.org/10.1016/j.eswa.2011.04.227

Dong, Y. C.; Xu, Y. F.; Li, H. Y. 2008. On consistency measures of linguistic preference relations, European Journal of Operational Research 189: 430-444. http://dx.doi.org/10.1016/j.ejor.2007.06.013

Herrera-Viedma, E.; Herrera, F.; Chiclana, F. 2002. A consensus model for multiperson decision making with different preference structures, IEEE Transactions on Systems, Man and Cybernetics, Part A 32: 394-402. http://dx.doi.org/10.1109/TSMCA.2002.802821

Hwang, C. L.; Yoon, K. 1981. Multiple attribute decision making: methods and applications. Berlin: SpringerVerlag. http://dx.doi.org/10.1007/978-3-642-48318-9

Li, D. F. 2010. A ratio ranking method of triangular intuitionistic fuzzy numbers and its application to MADM problems, Computers and Mathematics with Applications 60: 1557-1570. http://dx.doi.org/10.1016/j.camwa.2010.06.039

Li, D. F.; Chen, G. H.; Huang, Z. G. 2010. Linear programming method for multiattribute group decision making using IF sets, Information Sciences 180: 1591-1609. http://dx.doi.org/10.1016/j.ins.2010.01.017

Li, D. F.; Wang, Y. C.; Liu, S.; Shan, F. 2009. Fractional programming methodology for multi attribute group decision making using IFS, Applied Soft Computing 9: 219-225.

http://dx.doi.org/10.1016/j.asoc.2008.04.006

Liu, P. D. 2011. A weighted aggregation operators multi-attribute group decision-making method based on interval-valued trapezoidal fuzzy numbers, Expert Systems with Applications 38: 1053-1060. http://dx.doi.org/10.1016/j.apm.2011.09.006

Merigó, J. M.; Casanovas, M. 2010. Fuzzy generalized hybrid aggregation operators and its application in decision making, International Journal of Fuzzy Systems 12: 15-24.

Merigó, J. M.; Casanovas, M. 2011. The uncertain generalized OWA operator and its application to financial decision making, International Journal of Information Technology \& Decision Making 10(2): 211-230. http://dx.doi.org/10.1142/S0219622011004300

Merigó, J. M.; Gil-Lafuente, A. M. 2011. Fuzzy induced generalized aggregation operators and its application in multi-person decision making, Expert Systems with Applications 38: 9761-9772. http://dx.doi.org/10.1016/j.eswa.2011.02.023

Mohiuddine, S. A.; Şevli, H. 2011. Stability of Pexiderized quadratic functional equation in intuitionistic fuzzy normed space, Journal of Computational and Applied Mathematics 235: 2137-2146.

http://dx.doi.org/ 10.1016/j.cam.2010.10.010 
Mursaleen, M.; Mohiuddine, S. A. 2009. On lacunary statistical convergence with respect to the intuitionistic fuzzy normed space, International Journal of Approximate Reasoning 51: 56-70. http://dx.doi.org/10.1016/j.cam.2009.07.005

Nehi, H. M.; Maleki, H. R. 2005. Intuitionistic fuzzy numbers and it's applications in fuzzy optimization problem, in Proceedings of the 9th WSEAS international conference on systems, 2005, Athens, Greece.

Saadati, R.; Vaezpour, S. M.; Chao, Y. J. 2009. Quicksort algorithm: application of a fixed point theorem in intuitionistic fuzzy quasi-metric spaces at a domain of words, Journal of Computational and Applied Mathematics 228: 219-225. http://dx.doi.org/10.1016/j.cam.2008.09.013

Szmidt, E.; Kacprzyk, J. 2004. A similarity measure for intuitionistic fuzzy sets and its application in supporting medical diagnostic reasoning, Lecture Notes in Computer Science 3070: 388-393. http://dx.doi.org/10.1007/978-3-540-24844-6_56

Wang, J. Q.; Zhang, Z. 2009. Multi-criteria decision-making method with incomplete certain information based on intuitionistic fuzzy number, Control and Decision 24: 226-230.

Wang, Y. M.; Fan, Z. P. 2007. Fuzzy preference relations: aggregation and weight determination, Computers \& Industrial Engineering 53: 163-172. http://dx.doi.org/10.1016/j.cie.2007.05.001

Wang, Z. J.; Li, K. W.; Wang, W. Z. 2009. An approach to multiattribute decision making with intervalvalued intuitionistic fuzzy assessments and incomplete weights, Information Sciences 179: 3026-3040. http://dx.doi.org/10.1016/j.ins.2009.05.001

Wei, G. W. 2008. Maximizing deviation method for multiple criteria decision making in intuitionistic fuzzy setting, Knowledge-Based Systems 21: 833-836. http://dx.doi.org/10.1016/j.knosys.2010.01.003

Wei, G. W. 2009. Some geometric aggregation functions and their application to dynamic multiple attribute decision making in intuitionistic fuzzy setting, International Journal of Uncertainty, Fuzziness and Knowledge-Based Systems 17: 179-196. http://dx.doi.org/10.1142/S0218488509005802

Wei, G. W. 2010a. Some induced geometric aggregation operators with intuitionistic fuzzy information and their application to group decision making, Applied Soft Computing 10: 423-431.

http://dx.doi.org/10.1016/j.asoc.2009.08.009

Wei, G. W. 2010b. GRA method for multiple attribute decision making with incomplete weight information in intuitionistic fuzzy setting, Knowledge-Based Systems 23: 243-247. http://dx.doi.org/10.1016/j.knosys.2010.01.003

Wei, G. W. 2010c. Some arithmetic aggregation operators with intuitionistic trapezoidal fuzzy numbers and their application to group decision making, Journal of Computers 5: 345-351. http://dx.doi.org/10.4304/jcp.5.3.345-351

Wei, G. W. 2011. Gray relational analysis method for intuitionistic fuzzy multiple attribute decision making, Expert Systems with Applications 38: 11671-11677. http://dx.doi.org/10.1016/j.eswa.2011.03.048

Wei, G. W.; Zhao, X. F. 2012. Some induced correlated aggregating operators with intuitionistic fuzzy information and their application to multiple attribute group decision making, Expert Systems with Applications 39: 2026-2034. http://dx.doi.org/10.1016/j.eswa.2011.08.031

$\mathrm{Wu}$ J.; Cao, Q. W. 2011. Some issues on properties of the extended IOWA operators in fuzzy group decision making, Expert Systems with Applications 38: 7059-7066. http://dx.doi.org/10.1016/j.eswa.2010.12.007

Wu, J.; Cao, Q. W. 2013. Same families of geometric aggregation operators with intuitionistic trapezoidal fuzzy numbers, Applied Mathematical Modelling 37: 318-327. http://dx.doi.org/10.1016/j.apm.2012.03.001

Wu, J.; Chiclana, F. 2012. Non-dominance and attitudinal prioritisation methods for intuitionistic and interval-valued intuitionistic fuzzy preference relations, Expert Systems with Applications 39: 13409-13416. http://dx.doi.org/10.1016/j.eswa.2012.05.062

Wu, J.; Chiclana, F. 2014a. A social network analysis trust-consensus based approach to group decision-making problems with interval-valued fuzzy reciprocal preference relations, Knowledge-Based Systems 99: 97-107. http://dx.doi.org/10.1016/j.knosys.2014.01.017 
Wu, J.; Chiclana, F. 2014b. Visual information feedback mechanism and attitudinal prioritisation method for group decision making with triangular fuzzy complementary preference relations, Information Sciences 279: 716-736. http://dx.doi.org/10.1016/j.ins.2014.04.025

Wu, J.; Huang, H. B.; Cao, Q. W. 2013. Research on AHP with interval-valued intuitionistic fuzzy sets and its application in multi-criteria decision making problems, Applied Mathematical Modelling 37: 9898-9906. http://dx.doi.org/10.1016/j.apm.2013.05.035

Wu, J.; Li, J. C.; Li, H.; Duan, W. Q. 2009. The induced continuous ordered weighted geometric operators and their application in group decision making, Computers \& Industrial Engineering 56: 1545-1552. http://dx.doi.org/10.1016/j.cie.2008.09.041

Wu, J.; Liu,Y. J. 2013. An approach for multiple attribute group decision making problems with interval-valued intuitionistic trapezoidal fuzzy numbers, Computers \& Industrial Engineering 66: 311-324. http://dx.doi.org/10.1016/j.cie.2013.07.001

$\mathrm{Xu}, \mathrm{Z}$. S. 2005. Deviation measures of linguistic preference relations in group decision making, Omega 33: 249-254. http://dx.doi.org/10.1016/j.omega.2004.04.008

$\mathrm{Xu}, \mathrm{Z}$. S. 2009. An automatic approach to reaching consensus in multiple attribute group decision making, Computers \& Industrial Engineering 56: 1369-1374. http://dx.doi.org/10.1016/j.cie.2008.08.013

$\mathrm{Xu}, \mathrm{Z}$. S. 2010. A method based on distance measure for interval-valued intuitionistic fuzzy group decision making, Information Sciences 180(1): 181-190. http://dx.doi.org/10.1016/j.ins.2009.09.005

$\mathrm{Xu}$, Z. S. 2011. Approaches to multiple attribute group decision making based on intuitionistic fuzzy power aggregation operators, Knowledge-Based Systems 24(6): 749-760. http://dx.doi.org/10.1016/j.knosys.2011.01.011

Xu, Z. S.; Yager, R. R. 2009. Intuitionistic and interval-valued intutionistic fuzzy preference relations and their measures of similarity for the evaluation of agreement within a group, Fuzzy Optimization and Decision Making 8: 123-139. http://dx.doi.org/10.1016/j.apm.2013.01.044

Yager, R. R.; Filev, D. P. 1999. Induced ordered weighted averaging operators, IEEE Transactions on Systems, Man and Cybernetics 29: 183-190. http://dx.doi.org/10.1109/3477.752789

Ye, F. 2010. An extended TOPSIS method with interval-valued intuitionistic fuzzy numbers for virtual enterprise partner selection, Expert Systems with Applications 37: 7050-7055. http://dx.doi.org/10.1016/j.eswa.2010.03.013

Ye, J. 2011. Expected value method for intuitionistic trapezoidal fuzzy multicriteria decision-making problems, Expert Systems with Applications 38: 11730-11734. http://dx.doi.org/10.1016/j.eswa.2011.03.059

Zadeh, L. A. 1965. Fuzzy sets, Information and Control 8: 338-353. http://dx.doi.org/10.1016/S0019-9958(65)90241-X

Zhang, H. Y.; Zhang,W. X.; Wu, W. Z. 2009. On characterization of generalized interval-valued fuzzy rough sets on two universes of discourse, International Journal of Approximate Reasoning 51: 56-70. http://dx.doi.org/10.1016/j.ijar.2009.07.002

Zhang, X.; Liu, P. D. 2010. Method for aggregating triangular fuzzy intuitionistic fuzzy information and its application to decision making, Technological and Economic Development of Economy 16(2): 280-290. http://dx.doi.org/10.3846/tede.2010.18

Jian WU received the $\mathrm{PhD}$ degree in management science and engineering from Hefei University of Technology, Hefei, China, in 2008. He is an associated Professor with School of Economics and Management, Zhejiang Normal University, China. His current research interests include information fusion, group decision making, computing with words, and aggregation operators. He has published more than 30 papers in journals including International Journal of Intelligent Systems, Computers \& Industrial Engineering, Expert Systems with Applications, Applied Mathematical Modelling and Mathematical and Computer Modelling. 